\title{
The Beam-Forming Technique for Enhancement of Noisy Seismic Refraction Data
}

\author{
Arewa James Ogah ${ }^{1^{*}}$, Ani Donatus Chinedu ${ }^{2}$ \\ ${ }^{1}$ Department of Physics, Kogi State University, Anyigba, Nigeria \\ ${ }^{2}$ Department of Mathematics, Kaduna Polytechnic, Kaduna, Nigeria \\ Email: *afamogah@yahoo.com
}

Received July 14, 2012; revised August 17, 2012; accepted August 26, 2012

\begin{abstract}
We have described a method of obtaining useful information from noisy seismic refraction data. The simple method, tagged beam-forming technique, is based on the basic time-distance equations of refraction seismology. It involves basically of introducing relative time delays to individual seismic traces of seismic refraction spread to correct for the non-coincidence of the incidence seismic energy at different geophones, and averaging the traces to obtain the beam. The assumption here is that the signal is coherent between the geophones while the noise is random, and for groups of geophones corresponding to the same refraction segments of the travel time curve, this basic assumption is valid. The process of beam forming therefore leads to improvement in signal-to-noise ratio (SNR) and correct determination of the intercept times which are subsequently used to compute other geologic layer parameters. The ability of the applied technique to filter out or minimize random noise has been tested using a modified random number routine. The performance test on computation of geologic layer parameters using very noisy synthetic data reveals that the method is still very reliable even with very poor quality data having SNR as small as 0.05 .
\end{abstract}

Keywords: Seismic Refraction; Beam-Forming; Filter; Random Noise

\section{Introduction}

Interpretation of seismic data is often made tedious, and sometimes erroneous, because the seismic records contain not only primary events (or signal) from the subsurface interfaces but also spurious secondary events (or noise) which interfere with the desired signals. The on-set time can be badly obscured and picking of first arrivals become very uncertain if the energy of the signal relative to the energy of the noise (signal-to-noise ration) is low. Depending on their sources and their modes of propagations, such unwanted events can be coherent or incoherent [1-3]. The common causes of seismic noise are surface waves and near-surface waves (particularly Rayleigh waves) initiated by source pulses, multiple reflections between the surface and geologic interface, near-surface in-homogeneities such as pebbles, boulders, root of trees and other non-uniformity that can cause scattering of seismic waves locally. Such diverse types and varying frequencies of noise resulting from diverse sources require different approaches to remove or minimize their impacts on seismic interpretations.

Among different attempts made to reduce effects of random noise is the use of signal averaging system (SAS),

${ }^{*}$ Corresponding author. particularly when a low source of seismic energy is used [4]. The application of SAS involves multiple hammer blows for a particular geophone spread without removing the spread until the required number of blows has been taken [5]. For each of the geophones, the SAS averages the noise and the weak signals that arrive from all the hammer blows. This process, called "stacking”, enhances the weak primary events from the low energy source and suppresses the random noise. However, because each spread requires several hammer blows at the shot point, the method has the disadvantage of being slow with required increase labour and extra cost of purchasing additional component, the SAS machine.

Ervin et al. [6] used a digital computer method that automated the processing of refraction data by conventional multichannel seismic profile. Three different computer programs were used. The first, call PICK performed the most critical and difficult task of accurately determining the arrival time for each trace. The second, called VELDEP, translated the time-distant curves into velocities and depths while the third interpolated the depths at equal velocity intervals for display as a cross section printer plots. The PICK was used to stack corresponding traces from several shots thereby minimizing random noise on the stack traces. As emphasized by 
Ervin et al. [6], the algorithm does not permit application of dip correction and plotting of depths immediately beneath the shot points by VELDEP introduced some errors.

The technique of multi-refractor imaging was used by de Franco [7] to construct a single image of a number of geologic refractors. The method which improves signalto-noise ratio uses the first arrivals but without picking the arrival times. However, the approach is most effective with data that have been obtained with roll-along acquisition program which requires extra labour.

Most of the techniques thus discussed have been found to be successful, or at least capable of improving the record quality. In spite of these satisfactory performances, the limitations associated with them might be serious constraints in using them. A computer processing method called beam-forming technique which consists basically of introducing relative time delays to individual seismic traces of the seismic refraction spread to correct for the non-coincidence of the incidence seismic energy at the different geophones, and averaging the traces to obtain the beam, has some attractive features that make it applicable in processing noisy seismic refraction data. It is capable of determining layer velocities and intercept times automatically without having to use some of the special field and data processing techniques summarized above. The method does not need the time and the energy consuming multiple hammer blows as used with the signal averaging system. Although the proposed technique may not serve as a substitute to any of the techniques discussed earlier, it could be used as an alternative to a few of them. The primary objective of this study is the application of a suitable technique, the beam-forming technique, for the acquisition of accurate and reliable information from relatively noisy seismic refraction data.

\section{Methodology}

The beam-forming procedure uses the time-distance relation of refraction seismology in which the down-dip and the up-dip travel times [8], $t_{d}$ and $t_{u}$ respectively, for an $n$-th layer case can be expressed as

$$
t_{d n}=\frac{x}{v_{n}} \sin \left(i_{c}+\alpha\right)+2 \sum_{k=1}^{n-1} z_{d k} \frac{\sqrt{v_{n}^{2}-v_{k}^{2}}}{v_{k} v_{n}}
$$

and

$$
t_{u n}=\frac{x}{v_{n}} \sin \left(i_{c}-\alpha\right)+2 \sum_{k=1}^{n-1} z_{u k} \frac{\sqrt{v_{n}^{2}-v_{k}^{2}}}{v_{k} v_{n}}
$$

where $x$ is the distance of each geophone from the shot point; $v_{n}$ is the velocity of the $n$-th layer; $i_{c}$ is the critical angle of incidence at the lowest interface recorded by the geophones; $\alpha$ is the dip of the interface while $z_{d}$ and $z_{u}$ are the down-dip and up-dip perpendicular distances respectively between any two geologic interfaces. Equa- tions (1) and (2) can be re-expressed for each segment of the time-distance graph as

$$
\begin{gathered}
t_{d n}=\frac{x}{v_{n}} \sin \left(i_{c}+\alpha\right)+C_{d n} \\
t_{u n}=\frac{x}{v_{n}} \sin \left(i_{c}-\alpha\right)+C_{u n}
\end{gathered}
$$

where $C_{d n}$ and $C_{u n}$ are both zero for the over burden layer segment, but are down-dip and up-dip intercept times respectively on time axis for any other segment of the $t-x$ plot. The slope of each segment is given by either

$$
\frac{\sin \left(i_{c}+\alpha\right)}{v_{n}}=\frac{1}{v_{d}}
$$

or

$$
\frac{\sin \left(i_{c}-\alpha\right)}{v_{n}}=\frac{1}{v_{u}}
$$

where $v_{d}$ and $v_{u}$ are apparent velocities of the respective layers shooting down-dip and up-dip respectively. The intercept times can then be calculated from Equations (3) and (4) after substituting Equations (5) and (6); that is,

$$
\begin{gathered}
C_{d n}=t_{d n}-\frac{x}{v_{d}} \\
C_{u n}=t_{u n}-\frac{x}{v_{u}}
\end{gathered}
$$

\subsection{The Steering Delay Concept}

The travel time of seismic waves from shot point to each geophone increases with distance of the geophone away from the point (Figure 1(a)). To achieve the coherent arrangement of the traces refracted from the same layer (Figure 1(b)), appropriate values of the steering day term $\left(\frac{x}{v_{d}}\right.$ and $\frac{x}{v_{u}}$ in Equations (7) and (8)) computed for the individual trace is subtracted from the travel time $t$ of the corresponding trace. With the signal perfectly coherent along the refraction branch and the steering delays accurately determined, the energy of the averaged traces, the beam, is equal to the energy of the individual traces. This condition is achieved when the value of any of the apparent velocities in Equations (7) and (8) is equal to the refractor velocity. When the trial velocity is different from the refractor velocity, the traces are improperly steered and do not add up coherently when summed to get the beam. In this case, the beam energy is less than the energy of the individual traces.

The beam-forming technique is best understood when we represent the discrete amplitudes of the seismic traces as

$$
d_{i j}=s_{j}+\varepsilon_{i j} ; i=1,2, \cdots, K ; j=1,2, \cdots, L
$$

where $d_{i j}$ is the $j$-th sample of the trace at the $i$-th geophone; $s_{j}$ is the $j$-th sample of the signal; $\varepsilon_{i j}$ is the $j$-th 


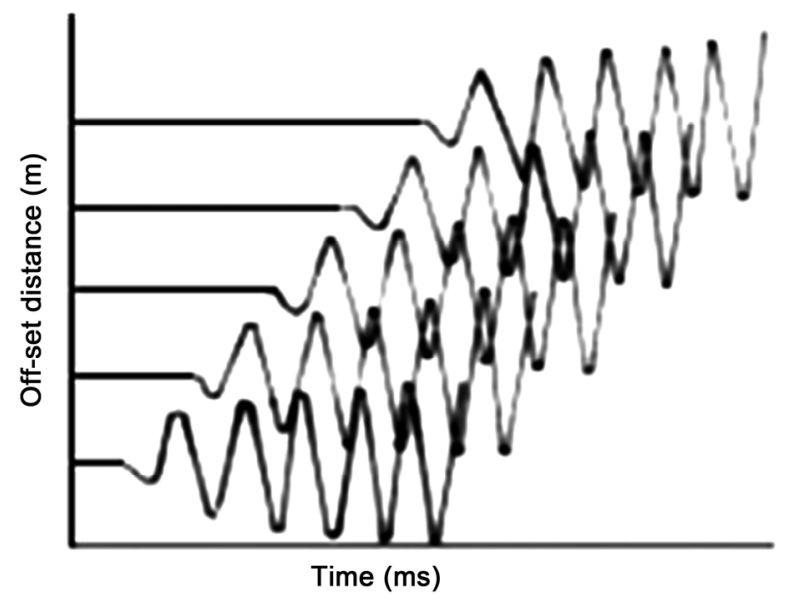

(a)

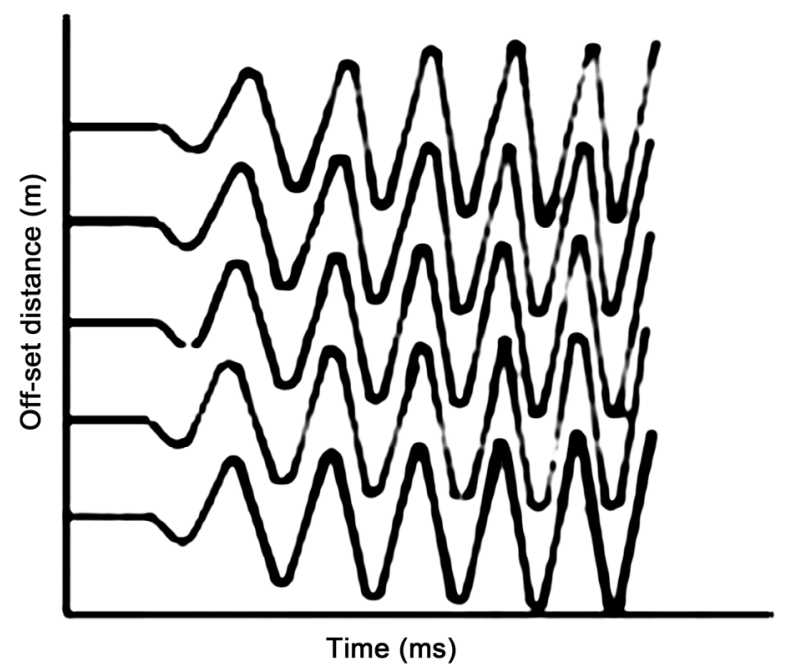

(b)

Figure 1. Simulating effect of steering delay on seismic traces from the same refractor: (a) Before the application; (b) After the application.

sample of the noise at the $i$-th geophone (assumed to be random); $K$ is the total number of traces summed to form the beam; while $L$ is the total number of sample points per trace. The equation of the beam is then given as

$$
B_{j}=\frac{1}{K} \sum_{j=1}^{L} d_{i j}
$$

That is,

$$
B_{j}=s_{j}+\frac{1}{K} \sum_{j=1}^{L} \varepsilon_{i j} \quad \text { (substituting Equation (10)) }
$$

If the noise is completely random, the noise term in Equation (11) vanishes and the beam is identical to the signal $s_{j}$. In the field situation, the $\varepsilon_{i j}$ cannot sum up to zero, but minimized to allow clear identification of the on-set time which is done by automatic picking. The energy of the beam $\left(E_{B}\right)$, defined as the sum of the squared amplitudes of the beam can then be expressed as

$$
E_{B}=\sum_{j=1}^{L} B_{j}^{2}
$$

For each trial velocity used, a beam is formed and the beam energy is calculated. The trial velocity which corresponds to the maximum beam energy is therefore the correct refractor velocity.

\subsection{Determination of Intercept Time}

The intercept time is related to the geologic layer thickness and velocities on the opposite sides of any interface. Therefore, correct determination of its value is paramount to obtaining good estimates of the depths of geologic interfaces and other layer parameters. Equations (7) and (8), relating the intercept time $C$ with other parameters are equations of the refraction branch of the $t-x$ plot in which $C$ is never equal to zero $[4,8]$. Therefore, the beam of maximum energy is of the form shown in Figure 2 in which the initial part is noisy, followed by the on-set of the signal at the arrowed point.

A FORTRAN subroutine has been written with the logic of picking the intercept time (the arrowed point) after scanning for the signal in the noisy part of the beam between the arrow and the point labelled A in Figure 2. Such value was then used to compute other geologic layer parameters.

\section{Performance Test on Noisy Synthetic Data}

In order to evaluate the performance of the applied technique in improving signal-to-noise ratio and hence, obtaining correct intercept times in noisy data of very low signal-to-noise ratio (SNR), we modified a random number routine [9] that was only designed to generate random numbers between 0 and 1 to generate both negative and positive random numbers of any desired amplitudes. These numbers were added to clean synthetic seismic traces to serve as noise. Using the simple geologic two layer model, the performance of the beam-forming technique was evaluated both qualitatively and quantitatively.

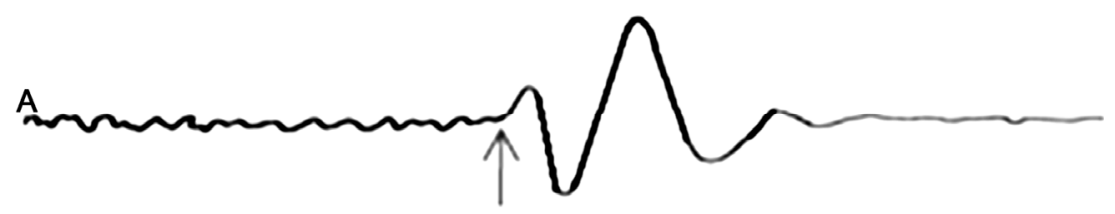

Figure 2. Sample of synthetic beam to illustrate the logic involved in the intercept time determination. 
The former was done using varying number of noisy traces which was increased in steps of two between two and eight traces per segment of the $t-x$ plot. In each case, the beam-forming technique was applied so as to estimate the randomness of the added noise and also to assess the qualities of the formed beams with increasing

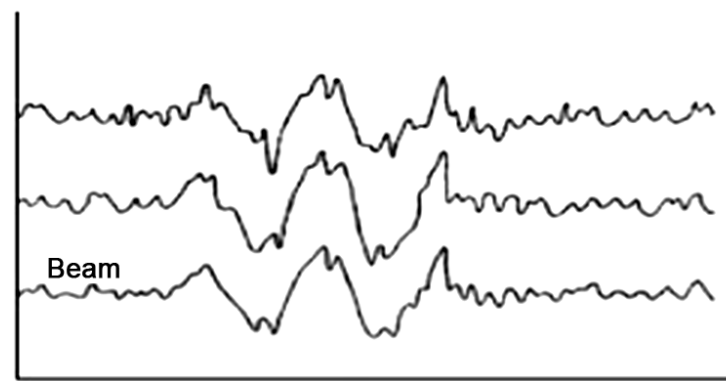

(a)

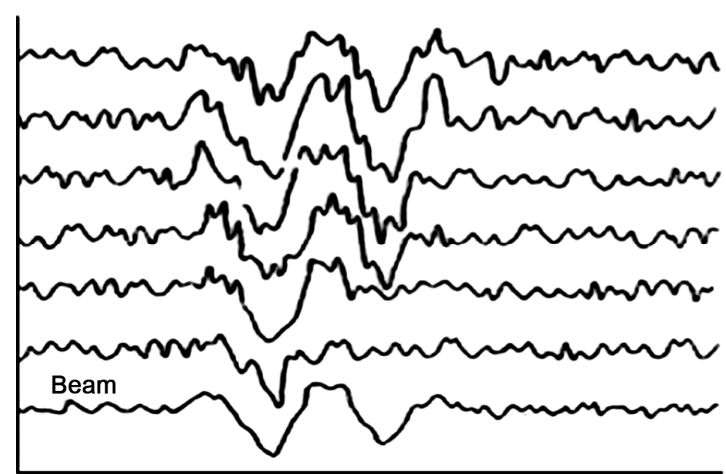

(c) number of traces per segment (Figure 3). For each set of noisy traces summed to form the beam, the SNR was computed (Table 1), the beam was plotted with the corresponding noisy traces (Figure 3 ), and the intercept times picked were used to compute other layer parameters (Table 2).

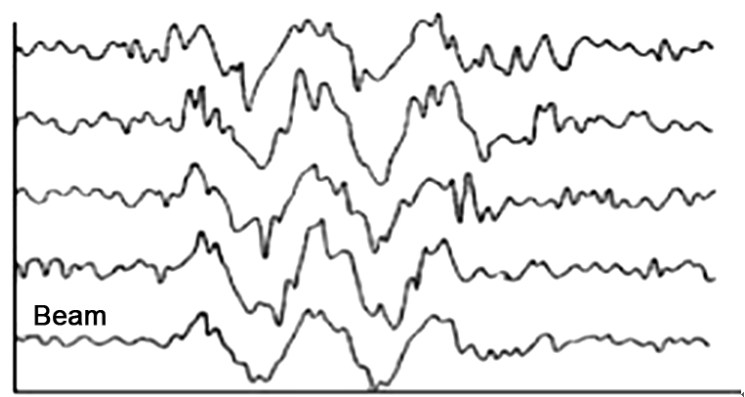

(b)

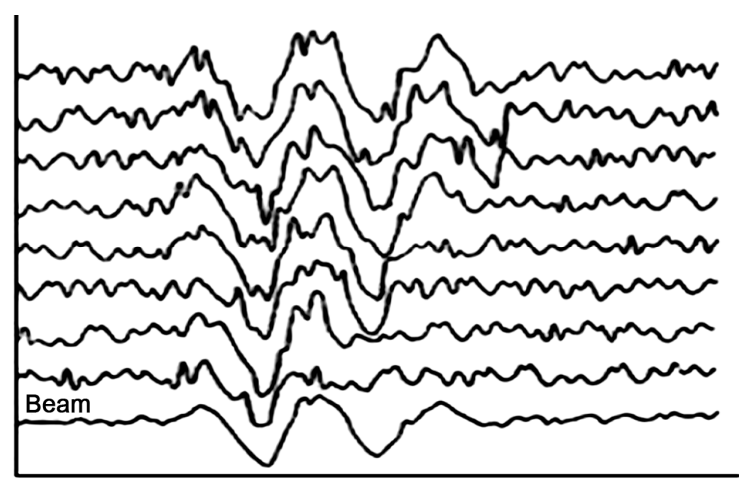

(d)

Figure 3. Assessing the randomness of the added noise and the qualities of the beam with increasing number of noisy traces.

Table 1. Signal-to-noise ratio improvement with number of traces.

\begin{tabular}{ccc}
\hline Serial No. & Number of summed traces & S/N improvement \\
\hline 1 & 2 & 1.41 \\
2 & 4 & 2.00 \\
3 & 6 & 2.45 \\
4 & 8 & 2.83 \\
\hline
\end{tabular}

Table 2. Quantitative assessment of the beam-forming technique using synthetic data.

\begin{tabular}{ccccccc}
\hline & $\begin{array}{c}\text { 2nd layer velocity } \\
(\mathrm{m} / \mathrm{s})\end{array}$ & $\begin{array}{c}\text { Relative } \\
\text { error (\%) }\end{array}$ & $\begin{array}{c}\text { Intercept } \\
\text { time (ms) }\end{array}$ & $\begin{array}{c}\text { Relative } \\
\text { error (\%) }\end{array}$ & $\begin{array}{c}\text { Depth of the } \\
\text { interface (m) }\end{array}$ & $\begin{array}{c}\text { Relative } \\
\text { error (\%) }\end{array}$ \\
\hline $\begin{array}{c}\text { Model parameters } \\
\text { Signal-to-noise ratio }\end{array}$ & 1000.00 & - & 10.00 & - & 3.00 & - \\
2.65 & 1010.00 & \pm 1.00 & 10.50 & \pm 5.00 & 3.02 & \pm 0.67 \\
0.68 & 1030.00 & \pm 3.00 & 10.80 & \pm 8.00 & 3.09 & \pm 3.00 \\
0.17 & 1040.00 & \pm 4.00 & 11.00 & \pm 10.00 & 3.14 & \pm 4.70 \\
0.05 & 1060.00 & \pm 6.00 & 12.40 & \pm 24.00 & 3.52 & \pm 17.33 \\
\hline
\end{tabular}


Quantitative assessment of the method was attempted by using model velocity values of $500 \mathrm{~ms}^{-1}$ and 1000 $\mathrm{ms}^{-1}$ for the first and second layers respectively. A first layer thickness of 3 metres was also chosen. A set of synthetic traces were then generated for the model. Various levels of noise were again added to the traces before the beam-forming technique was used to interpret the synthetic data. The deviations in values of parameters computed from those of the starting model as the SNR decreases was then considered a measure of the performance of the technique on synthetic data. Using these deviations, the relative percentage errors in each of the computed parameters was determined. Table 1 depicts the values of these parameters corresponding to different SNR and the relative percentage errors in each of the parameters.

\section{The Results}

The first assessment made was that of randomness of the applied noise. The results obtained and displayed in Table 1 indicate SNR improvement with number of summed traces $K$. It is obvious from the table that for any number of traces $K$, there is $K^{1 / 2}$ SNR improvement (gain in amplitudes of the signal over those of noise). This consistent variation agrees with the statistical test of randomness $[4,10]$ that if the numbers are truly random, the sum of $K$ random traces will be proportional to $K^{1 / 2}$, so that the SNR will be improved by $K^{1 / 2}$. Therefore the noise traces used are completely uncorrelated.

The quality of the beam signal, the end product of the applied steering delays and summation operations performed on the noisy data, determines the accuracy of the end results. A noise-free beam does not only indicate high improvement in the signal-to-noise ratio but leads to determination of the correct value of the intercept time which also yields correct values of other layer parameters. Figures 3(a) through (d) show the gradual improvement in the quality of the beams with increasing number of traces summed to form them. When the number of traces involved in a stack is small; e.g., 2 and 4 noisy traces in Figures 3(a) and (b), the beams are noisy and are almost identical to each of the traces. As the number of traces per stack increases (Figures 3(c) and (d)), the differences between the beams and the individual traces become obvious. The implication of that is if few receivers record arriving waves from a refractor, the determination of the intercept times will often be erroneous as a result of poor quality of the formed beams. That is, more reliable results are expected from closely planted geophones than those planted coarsely in a geologically noisy environment, or when low sources of seismic energy are used.

Table 2 shows that as the noise level increased, the values of the layer parameters (velocity and depth) com- puted progressively deviated from those of the model values. That is, the errors in the computed parameters increased with decreasing SNR. As depicted by the table, the fall in SNR from 2.65 to as low as 0.05 only resulted corresponding error of $1 \%$ to $6 \%$ in the refractor velocity and error of $0.67 \%$ to $17.33 \%$ in the depth of the geologic interface. The results of the qualitative analysis therefore show that the results of the beam-forming technique can be reliable even in an environment of very low signal-to-noise ratio.

\section{Discussions and Conclusion}

If an appreciable improvement in SNR is to be achieved when using low sources of seismic energy in a relatively noisy environment without the use of signal enhancement systems, some form of processing is essential. In this study, some causes of seismic noise and the abilities of various types of field techniques and digital processing methods have been reviewed. This was done to assess the level of SNR improvement so far achieved with the known methods, and how these improvements correlate with that attainable using the beam-forming technique.

The automatic procedure for the processing of lowquality seismic refraction data presented in this work include automatic determination of layer velocities and the corresponding intercept time which are used to compute other layer parameters. Though it is the basic concepts of the method and some few examples that we have presented here, a computer program based on this procedure has been written and tested on a large amount of data including noiseless and noisy synthetic data. We intentionally ignored the results obtained with the noiseless synthetic data since our target is the development of a technique for the acquisition of accurate and reliable results from relatively noisy data. The results with the noisy synthetic data has reviewed that our method of analysis is capable of determining depths with less than $20 \%$ error and velocity with less than $7 \%$ error even with almost uselessly-noisy data having SNR of about 0.05 or less. The beam-forming technique can therefore be applied in analysis of very noisy real refraction data but with the following limitations: 1) The method performs poorly in the presence of coherent noise; 2) Grouping of refraction signals from the same subsurface sources requires extra care as erroneous grouping of the traces will produce distorted beam that does not correspond to the correct seismic wave velocity for the layer. In search of a solution to the above problem, further studies are here recommended on how the beam-forming technique could be used to automatically determine the group of seismic traces that should belong to the same refractor by examing some properties of the individual traces. 


\section{REFERENCES}

[1] R. G. Anderson and G. A. McMechan, "Noise-Adaptive Filtering of Seismic Shot Records,” Geophysics, Vol. 53, No. 5, 1988, pp. 638-649.

[2] M. Bekara and M. van der Baan, "Random and Coherent Noise Attenuation by Empirical Mode Decomposition," Geophysics, Vol. 74, No. 5, 2009, pp. v89-v98.

[3] G. Liu, X. Chen, J. Du and K. Wu, "Radom Noise Attenuation Using f-x Regularized Non-Stationary AutoRegression,” Geophysics, Vol. 77, No. 2, 2012, pp. v61v69.

[4] W. M. Telford, L. P. Geldart and R. E. Sheriff, “Applied Geophysics,” 2nd Edition, Cambridge University Press, Cambridge, 1990.

[5] A. D. Chinedu and I. B. Osazuwa, "Generalized Reciprocal Method Applied in Processing Seismic Refraction Data from the Basement Terrain of Zaria, North-Western Nigeria,” Nigerian Journal of Physics, Vol. 20, No. 2,
2008, pp. 377-385.

[6] P. C. Ervin, L. D. McGinnis, R. M. Otis and K. C. Hall, "Automated Analysis of Marine Refraction Data, a Computer Algorithm,” Geophysics, Vol. 48, No. 5, 1989, pp. 582-589.

[7] R. De Franco, "Multi-Refractor Imaging with Stacked Refraction Convolution Section," Geophysical Prospecting, Vol. 53, No. 3, 2005, pp. 335-348. doi:10.1111/j.1365-2478.2005.00478.x

[8] W. Lowrie, "Fundamentals of Geophysics," Cambridge University Press, Cambridge, 1997.

[9] M. J. Merchant, "The ABC's of Fortran Programming," Wadsworth Publishing Co., California, 1979.

[10] D. Denham, “The Use of geophone Groups to Improve the Signal-to-Noise of the First Arrival in Refraction Shooting," Geophysical Prospecting, Vol. 11, No. 4, 1963, pp. 389-395. doi:10.1111/j.1365-2478.1963.tb02044.x. 